EPJ Web of Conferences 41, 04010 (2013)

DOI: $10.1051 /$ epjconf/20134104010

C Owned by the authors, published by EDP Sciences, 2013

\title{
Ultrafast Charge Separation in Low Band-Gap Polymer Blend for Photovoltaics
}

\author{
Margherita Maiuri ${ }^{1}$, Giulia Grancini ${ }^{2}$, Daniele Fazzi ${ }^{2}$, Hans-J. Egelhaaf ${ }^{3}$, Daniele Brida ${ }^{1}$, Giulio \\ Cerullo $^{1}$, and Guglielmo Lanzani ${ }^{1,2}$ \\ ${ }^{1}$ IFN-CNR, Dipartimento di Fisica, Politecnico di Milano, Piazza L. da Vinci, 32, 20133 Milano, \\ Italy. \\ ${ }^{2}$ Center for Nano Science and Technology @Polimi Istituto Italiano di Tecnologia, Via Pascoli 70/3 \\ 20133 Milano, Italy. \\ ${ }^{3}$ Konarka Technologies GmbH, Landgrabenstrasse 94, 90443 Nürnberg, Germany
}

\begin{abstract}
We track ultrafast charge dissociation in a particularly promising low-bandgap-polymer:fullerene blend for organic photovoltaics. Impulsive photoexcitation with excess energy leads to a 30 -fs formation of an hot charge transfer state, precursor of free carriers.
\end{abstract}

A new class of promising low band gap polymers is emerging for efficient organic photovoltaics (OPV). Among these, the poly[2,6-(4,4-bis-(2-ethylhexyl)-4Hcyclopenta[2,1-b;3,4-b']-dithiophene)alt-4,7-(2,1,3-benzothiadiazole)] (PCPDTBT) offers significative advantages in terms of lightharvesting and efficient charge transport properties [1]. When blended with the electron acceptor fullerene-derivative [6,6]-phenyl C61-butyric acid methyl ester (PCBM), these low-band-gap polymers allow the achievement of record-high efficiencies towards $10 \%$ in OPV [2] because they can harvest a broader portion of the visible light spectrum.

The time scale for charge separation at an electron donor/acceptor interface strongly influences the fundamental photovoltaic process, thus affecting the performance of OPV cells. Indeed, the formation of charge transfer (CT) state from higher excited electronic states competes with internal conversion on a time scale faster than $100 \mathrm{fs}[3,4]$. On the other hand, excess energy influences these phenomena, leading to $i$ ) additional energy dissipation, if relaxation is faster than charge separation; or ii) ultrafast formation of an hot CT state in the opposite case.

So far, experiments have not provided a clear evidence of these processes, due to their limited temporal resolution ( $\sim 150$ fs) [2,3]. Here, we exploit our tunable sub-20 fs pump-probe system [5] to observe in real time the CT state formation at the PCPDTBT:PCBM interface, by exciting the system with different photon energies. The time resolved experiments are supported by quantum chemical modelling of the interfacial states [6]. We show that photon excitation with excess energy with respect to the polymer gap leads to hot injection from high polymer excited states to high lying (hot) interfacial charge transfer states. From transition density matrix calculations we obtain that hot CT states are strongly delocalized resulting in an enhanced dissociation probability.

We investigate the photophysics of both the pristine polymer and the PCPDTBT:PCBM blends, by resonantly exciting the first singlet state $\left(S_{1}\right)$ at $710 \mathrm{~nm}$ and higher energy states $\left(S_{n}\right)$ at $510 \mathrm{~nm}$. Fig. 1 shows the two-dimensional differential transmission $\Delta \mathrm{T} / \mathrm{T}(\lambda, \tau)$ maps as a function of probe wavelength and pump-probe delay.

This is an Open Access article distributed under the terms of the Creative Commons Attribution License 2.0, which permits unrestricted use, distribution, and reproduction in any medium, provided the original work is properly cited. 


\section{$\lambda$ Pump: $710 \mathrm{~nm}$}

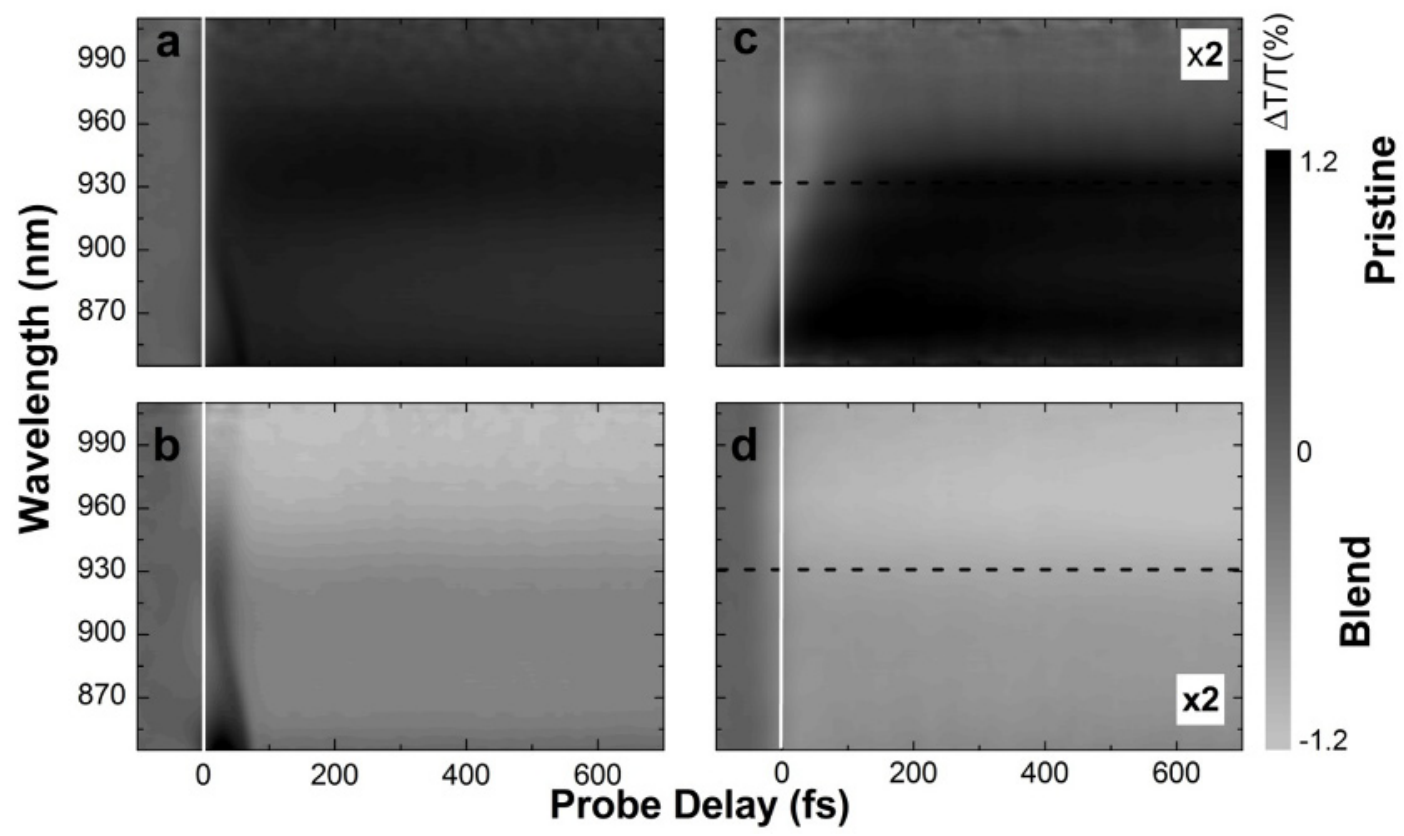

\section{$\lambda$ Pump: $510 \mathrm{~nm}$}

Fig. 1. 2D pump-probe maps as a function of the probe wavelength and pump-probe delay upon excitation at: (a, b) $710 \mathrm{~nm}$ pump wavelength, resonant to the $S_{1}$ state of the polymer. (c, d) $510 \mathrm{~nm}$ pump wavelength, resonant to the $S_{n}$ state of the polymer. The horizontal dashed lines indicates the probe wavelength corresponding to dynamics presented in Fig. 2.

The pristine polymer (Fig.1(a,c)) exhibits, in the probe spectral region, a broad positive signal assigned to stimulated emission (SE) (dark in Fig 1(a,c)) from $S_{1}$ to the ground state. We observe that, upon $\mathrm{S}_{\mathrm{n}}$ excitation, a weak photoinduced absorption (PA) band around $1000 \mathrm{~nm}$, due to transition to higher singlet states, is also revealed at early times. Instead, in PCPDTBT/PCBM blend the $\mathrm{SE}$ band is strongly suppressed if $\mathrm{S}_{1}$ is excited (Fig. 1(b)) and totally quenched upon $\mathrm{S}_{\mathrm{n}}$ excitation (Fig. 1(d)) by the formation of a broad PA band. This new band, assigned to transitions within the $\mathrm{CT}$ states manifold $\left(\mathrm{CT} \rightarrow \mathrm{CT}^{*}\right)$ in agreement with the theoretical picture, provides a spectral fingerprint for the $\mathrm{CT}$ state formation process.

Fig. 2(a) shows the $\Delta \mathrm{T} / \mathrm{T}$ time traces at a selected probe wavelength $(930 \mathrm{~nm})$ with $510-\mathrm{nm}$ pump excitation. In neat PCPDTBT (red line) the dynamics shows the initial PA giving way to the recovered SE within about 200 fs. In the blend (black line) we clearly resolve the ultrafast formation of the CT absorption band with a rate $k_{n}=1 / \tau_{n} \sim(30 \mathrm{fs})^{-1}$. These values govern the branching ratio between $S_{n} \rightarrow S_{1}$ and $S_{n} \rightarrow C T$ conversion processes, as schematically depicted in Fig. 2(b) where both possible pathways for the pristine polymer (left side) and for the blend (right side) cases are reported. We observe that the path to CT becomes highly favorable in case of high energy photoexcitation by comparing $k_{n}$ with the rate rate $k_{1}=1 / \tau_{1} \sim(45 \mathrm{fs})^{-1}$ measured for direct $S_{1}$ photoexcitation. This result is validated by quantum chemical calculations of the electronic couplings $\left(V_{i-j}\right)$ : the values for $\mathrm{S}_{\mathrm{n}}-\mathrm{CT}^{*}$ coupling $\left(V_{S n-C T^{*}}=0.1 \mathrm{eV}\right)$ are higher than for $\mathrm{S}_{1}-\mathrm{CT}_{1}\left(V_{S I-}\right.$ $\left.{ }_{C T I}=0.06 \mathrm{eV}\right)$. Moreover, at high energies, the calculated density of states $(\rho(\mathrm{DOS}))$ for CT excited states is larger. Both these observations suggest, according to the generalized Fermi golden rule $\left(k_{i-j}\right.$ $\left.\propto V_{i-j} \cdot \rho(\mathrm{DOS})\right)$, that the $\mathrm{S}_{\mathrm{n}} \rightarrow \mathrm{CT}_{\mathrm{n}}$ transfer rate is higher with respect to $\mathrm{S}_{1} \rightarrow \mathrm{CT}_{1}$. In addition, the high energy CT states delocalization leads to an easier formation of separated charges. This explains the higher external quantum efficiency (EQE) measured in the blue wavelength region (Fig. 2(c)) with respect to the absorption spectrum of the blend. 

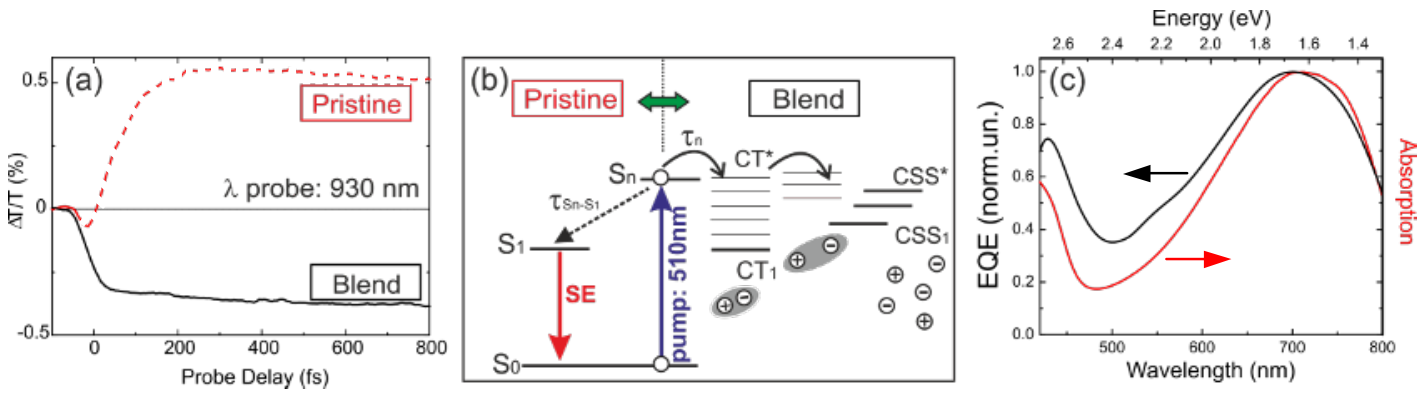

Fig. 2. (a) Pump-probe time traces at $930 \mathrm{~nm}$ probe wavelength for pristine PCPDTBT (dashed line) and blend PCPDTBT:PCBM (solid line) upon photoexcitation at 510-nm. Pump Intensity: $10 \mu \mathrm{J} / \mathrm{cm}^{2}$; (b) sketch of the energy level scheme when pumping $S_{n}$ in both pristine and blend systems: in the pure polymer (left panel) energy relaxation occurs by internal conversion to the $S_{1}$ state $\left(k_{\mathrm{Sn}-\mathrm{S} 1}=1 / \tau_{\mathrm{Sn}-\mathrm{S} 1} \sim\right.$ $\left.(140 \mathrm{fs})^{-1}\right)$, from which SE is probed at later times; in the blend (right panel) an ultrafast hot injection to an interfacial CT $*$ state prevails $\left(\mathrm{k}_{\mathrm{n}}=1 / \tau_{\mathrm{n}} \sim(30 \mathrm{fs})^{-1}\right)$, leading to weakly bound $\mathrm{CT}$ pairs that can more easily split into charge separated states (CSS); (c) EQE of the PCPDTBT:PCBM blend compared its absorption spectrum.

In conclusion, we have detected an ultrafast CT state formation at the interface between PCPDTBT donor polymer and PCBM acceptor. We find that charge separation is more efficient via hot dissociation through a $\mathrm{S}_{\mathrm{n}} \rightarrow \mathrm{CT}^{*}$ transfer mechanism. This gives an explanation for the faster CT state formation and the overall efficiency of the charge generation process. This work suggests that photovoltaic conversion via hot state dissociation in low-band gap polymer:PCBM blends can be an efficient route to be explored for efficiency optimization in OPV devices.

\section{References}

1. D. Mühlbacher, M. Scharber, M. Morana, Z. Zhu, D. Waller, R. Gaudiana, C. J. Brabec, Adv. Mater. 18, 2884 (2006).

2. Yang Yang of UCLA-Mitsubishi Chemical.

3. I. W.Hwang, Q. H Xu, C. Soci, B. Q. Chen, A. K. Y. Jen, D. Moses, A.J. Heeger, Adv. Funct. Mater. 17, 563 (2007).

4. F. Deschler, E. Da Como, T. Limmer, R. Tautz, T. Godde, M. Bayer, E. von Hauff, S. Yilmaz, S. Allard, U. Scherf, J. Feldmann, Phys Rev. Lett. 107, 127402 (2011).

5. D. Brida, C. Manzoni, G. Cirmi, D. Polli, G. Cerullo, IEEE J. Sel. Top. Quant. El. 18, 329 (2012).

6. G. Grancini, D. Polli, D. Fazzi, J. Cabanillas-Gonzalez, G. Cerullo, G. Lanzani, J. Phys. Chem. Lett. 2, 1099 (2011). 